Artigo Original

\title{
Dor lombar inespecífica e sua relação com o processo de trabalho de enfermagem*
}

\author{
Zulamar Aguiar Cargnin 1 \\ (iD) https://orcid.org/0000-0002-2731-5323 \\ Dulcinéia Ghizoni Schneider ${ }^{2}$ \\ (D) https://orcid.org/0000-0002-4842-2187 \\ Mara Ambrosina de Oliveira Vargas 2 \\ (1D) https://orcid.org/0000-0003-4721-4260 \\ Rosani Ramos Machado ${ }^{2}$ \\ (DD https://orcid.org/0000-0001-8287-4171
}

Objetivo: relacionar a dor lombar inespecífica dentro do contexto de trabalho da enfermagem com suas cargas de trabalho, processos de desgaste e os riscos de adoecimento. Método: estudo transversal com 301 trabalhadores de um hospital geral do Sul do país. Utilizaram-se o Nordic Musculoskeletal Questionnaire e a Escala de Avaliação do Contexto de Trabalho composta de três dimensões: "condições de trabalho", "organização do trabalho" e "relações socioprofissionais". Foi testada a associação das variáveis com a dor lombar por meio de análises bivariadas e multivariadas. A medida de associação utilizada foi a Odds Ratio e seus respectivos intervalos com 95\% de confiança. Os dados coletados foram discutidos sob o referencial teórico do processo de trabalho dentro da concepção marxista e da Teoria da Determinação Social do Processo Saúde-doença. Resultados: houve associação estatisticamente significativa entre as dimensões "organização do trabalho" e "condições de trabalho com a dor lombar", que obtiveram classificação crítica, significando riscos moderados ao adoecimento profissional. Conclusão: o estudo permitiu maior compreensão do processo de trabalho de enfermagem e sua relação com a lombalgia inespecífica e sinalizou que devem ocorrer mudanças na organização e nas condições de trabalho a fim de diminuir os riscos de adoecimento dos trabalhadores de enfermagem.

Descritores: Dor Lombar; Processo de Enfermagem; Condições de Trabalho; Saúde do Trabalhador; Carga de Trabalho; Enfermagem.

\section{Como citar este artigo}

Cargnin ZA, Schneider DG, Vargas MAO, Machado RR. Non-specific low back pain and its relation to the nursing work process. Rev. Latino-Am. Enfermagem. 2019;27:e3172. [Access DOI: http://dx.doi.org/10.1590/1518-8345.2915.3172.

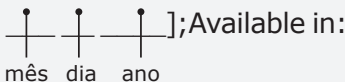

mês dia ano
URL 


\section{Introdução}

O trabalho é um intercâmbio entre o homem e a natureza que transforma e exprime uma finalidade. Ao mesmo tempo, pode ocorrer a exploração da força de trabalho, por meio de sua organização e divisão, cujos fatores econômicos exercem influência positiva ou negativa ${ }^{(1-2)}$. É necessário entender o caráter social do binômio saúde-doença em conexão com o meio/sistema de produção. O processo laboral é, ao mesmo tempo, social e biopsíquico. Identificar a causa dos agravos nesse cenário constitui-se de uma prática social em que se buscam, na coletividade, fatores e determinantes responsáveis pelo perfil de adoecimentos dos trabalhadores ${ }^{(3-4)}$.

Os profissionais, que atuam na área da enfermagem, expõem-se a diferentes cargas que comprometem sua vida e saúde ${ }^{(4)}$. Essas cargas interagem entre si e com o corpo do trabalhador, o qual estabelece respostas ao processo global laboral(5-6). Então, podem advir doenças ocupacionais, desgastes, absenteísmos e acidentes que geram custos financeiros e diminuição da produtividade, qualidade e segurança da assistência ${ }^{(4,7)}$. A enfermagem é foco de pesquisas, principalmente em nível hospitalar, devido às condições adversas de suas atividades e à exposição a várias cargas que se inter-relacionam entre si e com as formas de organização das tarefas ${ }^{(8-9)}$.

A dor lombar ou lombalgia está localizada na região lombar, entre o último arco costal e a prega glútea(10). Por sua vez, é inespecífica, quando não possui um diagnóstico específico e bem determinado, além de corresponder a cerca de 90 a 95\% dos $\operatorname{casos}^{(11-12)}$. Enquadra-se em um agravo ocupacional que se constitui um problema de saúde pública mundial devido à sua alta prevalência. A mesma atinge todas as faixas etárias e níveis socioeconômicos e requer promoção, educação e prevenção, não somente a reabilitação da saúde, com iniciativas globais eficazes(11-12).

Os fatores relacionados à dor lombar são múltiplos e complexos ${ }^{(11-13)}$. Os riscos profissionais envolvem a visão do contexto do trabalho, das demandas físicas, dos fatores ergonômicos, psicossociais e das formas de organização e execução das tarefas ${ }^{(13)}$

Este estudo objetivou relacionar a dor lombar inespecífica dentro de um contexto de trabalho da enfermagem com suas cargas de trabalho, processos de desgaste e os riscos de adoecimento. Para isso, analisou-se o inter-relacionamento de aspectos do processo laboral envolvendo suas condições, organização e relações socioprofissionais.

\section{Método}

Estudo de corte transversal realizado em um hospital público de média e alta complexidade de Florianópolis, vinculado à Secretaria Estadual de Saúde, com especialidades diversas. No momento da coleta de dados, possuía 225 leitos ativos. O local foi escolhido devido à multiplicidade de especialidades, além de possuir um contingente expressivo de profissionais de enfermagem.

Foi discutido sobre o referencial teórico da concepção marxista(1) $^{(1)}$ e a Teoria da Determinação Social do Processo Saúde-doença baseada nas cargas de trabalho, a partir de um estudo de $1989^{(3-4)}$. Ambas foram pautadas no materialismo histórico e dialético que reflete a realidade e a dinâmica social para explicar o processo saúde-doença, interligando, neste caso, a lombalgia inespecífica. Considera-se que o processo de trabalho e as forças produtivas influenciam o perfil patológico da enfermagem, já que esta profissão está exposta a diferentes cargas laborais. Estas, para este estudo, são definidas como o inter-relacionamento de fatores com o corpo do trabalhador que provocam desgaste e dizem respeito às condições, organização e divisão laboral que permeiam o trabalho assistencial(4,14). As cargas laborais podem ser agrupadas em físicas, químicas, biológicas e mecânicas, que possuem materialidade externa, observadas diretamente. Como, também, podem ser divididas em fisiológicas e psíquicas, que são de materialidade interna porque só adquirem materialidade no corpo humano por meio de transformações nos seus processos internos e manifestam-se por meio de distúrbio ou doença ${ }^{(4,6-7,14)}$.

Foram convidados a participar do estudo todos os auxiliares, técnicos de enfermagem e enfermeiros que se encontravam em atuação no hospital, no período da coleta de dados, caracterizando um censo de 353 trabalhadores. Após aplicar os critérios de inclusão e exclusão, a amostra final foi constituída de 301 trabalhadores de enfermagem, caracterizando $87,8 \%$ da população convidada a participar do estudo.

Foram incluídos profissionais que trabalham exclusivamente na enfermagem e que exercem por, pelo menos, um ano suas atividades. Esse tempo foi selecionado para estabelecer um possível nexo com a atividade laboral como contribuinte para a dor lombar. Excluíram-se os portadores de lombalgia com diagnósticos específicos como espondilolistese, hérnia de disco, estenose de canal medular, doenças infecciosas de coluna, tumores de coluna e fraturas.

A coleta de dados foi realizada no período de maio a outubro de 2017. Os trabalhadores foram abordados individualmente em seu turno de trabalho, em todos os setores do hospital, utilizando-se as escalas de serviço, abrangendo os turnos da manhã, tarde e noite. A cada entrega do formulário de perguntas autoaplicado, eram explicados os objetivos da pesquisa, a sua importância, o sigilo das informações, o anonimato dos participantes, instruções de preenchimento e, mediante a participação da pesquisa, foi agendada uma data para a devolução e validação dos questionários pelos participantes. 
O levantamento de dados foi realizado por meio de um formulário multidimensional, com questões abertas e fechadas relacionadas a dados sociodemográficos e laborais, construído pelas autoras; o Nordic Musculoskeletal Questionnaire (NMQ) e a Escala de Avaliação do Contexto de Trabalho (EACT).

A variável dependente foi a dor lombar extraída do NMQ, autorrelatada e identificada por uma figura que especificava o local para a sua identificação. As variáveis independentes foram sexo, idade (quantitativa contínua), categorizada por faixa etária, estado conjugal, número de filhos, cargo, turno de trabalho, anos de trabalho, hora plantão, outro vínculo empregatício e Índice de Massa Corpórea (IMC); condições ambientais de trabalho satisfatórias e insatisfatórias que englobam temperatura, espaço físico, mobiliário, higiene, instalações sanitárias, local para repouso e alimentação, acidentes de trabalho, sensação de sobrecarga, mau humor, cansaço ou fadiga ao final da jornada de trabalho; fatores que causam insatisfação no trabalho como a falta de reconhecimento, falta de segurança no emprego, impossibilidade de crescimento profissional, falta de autonomia, ambiente de trabalho ruim, trabalho que realiza, relacionamento com a chefia, falta de treinamento, sobrecarga de trabalho, instalações inadequadas (banheiros, vestiários, etc.) e salário. Para a determinação do IMC, usaram-se o peso e a estatura autorreferidos, classificados em baixo/adequado $\left(<25 \mathrm{Kg} / \mathrm{m}^{2}\right.$ ), sobrepeso ( $\geq 25$ e $<30$ $\mathrm{Kg} / \mathrm{m}^{2}$ ) e obesidade $\left(\geq 30 \text { e } \geq 40 \mathrm{Kg} / \mathrm{m}^{2}\right)^{(15)}$.

O NMQ identifica a dor ou o desconforto musculoesquelético nos últimos doze meses, bem como nos últimos sete dias, nas áreas anatômicas mostradas por uma figura. A versão brasileira(16) apresenta boa confiabilidade e os valores do coeficiente de Kappa foram de, no mínimo, 0,75 para cada item do questionário. Constitui-se de um importante instrumento para a identificação das dores mais prevalentes, da área anatômica específica e da incapacidade na realização de atividades. A variável "dor lombar" foi dicotomizada com base nas respostas agrupadas do NMQ e significou não (não e raramente) e sim (frequentemente e sempre). Escolheu-se esse instrumento por ser utilizado mundialmente em estudos epidemiológicos com várias populações, inclusive da enfermagem, além de ser simples e de fácil aplicação. É uma forma de padronização entre as pesquisas e favorece a comparação dos resultados.

A EACT foi desenvolvida em 2003 e validada posteriormente entre os anos de 2004 e 2006. Esta escala faz um levantamento das percepções que as pessoas fazem do seu contexto de trabalho, ressaltando os pontos críticos $^{(17)}$. A base da escala é o conceito de Contexto de Produção de Bens e Serviços, que une múltiplas e diversificadas variáveis em uma totalidade e faz um diagnóstico organizacional(18). A mesma analisa três dimensões interdependentes, que são "a organização do trabalho", "as condições de trabalho" e as "relações socioprofissionais". A dimensão "condições de trabalho" é expressa pela qualidade do ambiente físico, posto de trabalho, equipamentos e material disponibilizado para a execução do trabalho, sendo composta por dez itens. A dimensão "organização do trabalho" expressa a divisão das tarefas, normas, controles e ritmos de trabalho, tendo 11 itens. A dimensão "relações socioprofissionais" expressa os modos de gestão de trabalho, comunicação e interação profissional, sendo composta por dez itens. As respostas utilizam uma escala tipo Likert que vai de um (nunca) a cinco (sempre) ${ }^{(17-18)}$.

O banco de dados foi formado no programa Microsoft Excel. As variáveis sofreram análises descritivas. Já as categóricas foram submetidas à análise de frequências absoluta e relativa e, por fim, as contínuas, pelas médias e desvio-padrão (DP). A análise foi realizada no programa Statistical Package for the Social Sciences (SPSS), versão 23.0 (IBM Corp., Armonk, Estados Unidos). Foram aplicados o teste qui-quadrado com dicotomização das variáveis categóricas e o cálculo da medida de associação do Odds Ratio (OR) e respectivo intervalo de $95 \%$ de confiança (IC 95\%). Essa associação também foi feita na regressão logística binária nas análises bruta e ajustada. $O$ valor de $p$ utilizado foi $p<0,05$. As variáveis de confusão foram selecionadas a partir da literatura, por apresentarem relação com o desfecho. O método de entrada das variáveis no modelo de regressão foi o método ENTER ("entrada forçada"). A significação do modelo foi realizada pelos testes Omnibus $(p<0,05)$ e a qualidade, pelo teste de Hosmer e Lemeshow ( $p>0,05)$, e os valores da análise dos resíduos estavam dentro do intervalo de $\pm 2,5$. Também foram verificados a distância de Cook e o DFBeta para constante, para verificar casos possivelmente influentes (considerando valores maiores que um). Os fatores de confundimento considerados foram ajustados por faixa etária, sexo, estado conjugal, turno, cargo, anos de trabalho, Índice de Massa Corpórea, hora plantão, outro vínculo.

$\mathrm{Na}$ análise de dados da EACT, calcula-se a soma dos valores atribuídos a cada item para obter a média. $A$ interpretação dos resultados baseou-se na classificação de risco de adoecimento estabelecida pelos autores do instrumento como: satisfatório $(1-2,3)$, crítico $(2,4-3,7)$ e grave $(3,8-5,0)^{(17-18)}$. O grau satisfatório traduz um resultado positivo e de bem-estar no trabalho, sendo este um aspecto que deve ser mantido e consolidado no ambiente organizacional. O grau crítico demonstra um resultado mediano, indicando uma situação-limite, evidenciando um mal-estar no trabalho e risco de adoecimento. Já o grau grave mostra um resultado negativo e indica mal-estar no trabalho. Assim, existe um forte risco de adoecimento e requerem-se providências imediatas nas causas dos agravos, visando a eliminá-las e/ou a atenuá-las(17). 
O projeto de pesquisa obteve aprovação do Comitê de Ética em Pesquisa envolvendo Seres Humanos sob Parecer número 2.081.192/2017 e CAAE. 64164717.1.0000.0121.

\section{Resultados}

Em relação às características sociodemográficas, o sexo feminino predominou, sendo $83,4 \%$ da amostra; $66,4 \%$ são casados ou vivem com companheiros e $68,1 \%$ possuem filhos. O IMC médio foi de $26,34 \mathrm{~kg} / \mathrm{m}^{2}$ (DP 4,54), com um mínimo de 17,97 e o máximo de 46,71 , correspondendo à categoria de sobrepeso, segundo a Organização Mundial da Saúde (OMS), para ambos os sexos. Pouco mais da metade $(52,5 \%)$ está acima do IMC considerado normal. A categoria sobrepeso correspondeu a $34,6 \%$ da amostra, apesar de ter um predomínio de $41,9 \%$ com peso normal. A média de idade foi de 41,12 anos (DP 8,94), com o mínimo de 22 anos e o máximo de 64 anos (moda 38 anos). Quando categorizada por faixa etária, mostrou-se um percentual de 30 anos ou mais (10\%), 31 anos ou mais (34,5\%), 41 a 50 anos $(32,2 \%)$ e 51 ou mais $(23,3 \%)$.

Quanto às variáveis laborais, a categoria auxiliar/ técnico de enfermagem correspondeu a $79,4 \%$ e os enfermeiros somaram 20,6\%. Em relação aos anos de trabalho, a maior frequência $(36,5 \%)$ foi de um a quatro anos. A maioria dos participantes $(78,4 \%)$ faz turnos de 12 horas, sendo $46,2 \%$ durante o dia e $31,6 \%$ à noite; $76,4 \%$ fazem hora plantão e $72,1 \%$ não têm outro vínculo empregatício. A jornada de trabalho na instituição é de 30 horas/semanais, no entanto, se considerar aqueles que fazem hora plantão (hora extra), a jornada aumenta para uma média de 50 horas semanais.

Prevalência de dor ou desconforto na região lombar, nos últimos 12 meses $(51,4 \%)$ e na última semana $(45,4 \%)$, alcançou os maiores índices em relação às outras regiões do corpo e foi seguida pelo ombro $(46,1 \%)$, região cervical, com 40,9\%, e quadril (39,7\%), nos últimos 12 meses; região cervical (40,3\%), ombros $(34,7 \%)$ e quadril $(33,3 \%)$, na última semana. Aproximadamente $85 \%$ dos trabalhadores relataram ter, pelo menos, um sintoma musculoesquelético. De maneira geral, a prevalência de dor em outras regiões também foi bastante reportada. Quanto à limitação das atividades de vida diária (AVD), consequente do sintoma musculoesquelético na região lombar, nos últimos 12 meses, 81,9\% não apresentaram limitação; ainda assim, a região em que houve maior limitação foi a lombar $(18,1 \%)$, seguida pelo quadril $(14,1 \%)$ e região cervical $(14,1 \%)$.

$\mathrm{Na}$ análise das condições ambientais do seu local de trabalho, classificadas em sim (satisfatórias) ou não (insatisfatórias), a maioria considerou como insatisfatórios quase todos os itens: temperatura $(66,2 \%)$; espaço inapropriado (71\%); mobiliário $(78,5 \%)$; instalações sanitárias $(78,2 \%)$; repouso $(85 \%)$; exceto as condições de higiene que um pouco mais da metade dos respondentes $(52,5 \%)$ considerou satisfatórias. Cerca de $48 \%$ dos trabalhadores já apresentaram algum acidente de trabalho, sendo o mais comum com objeto perfurocortante. A maioria dos entrevistados não reconhece os riscos no local de trabalho ou não respondeu. Entre as sugestões dos participantes para melhorar as condições de trabalho estava, principalmente, aumentar o efetivo de recursos humanos.

Ao responder ao questionamento de como o trabalhador se sente ao final da jornada, variável também classificada como sim ou não, o fato de se sentir sobrecarregado $(p=0,001)$, mal-humorado $(p=0,000)$ e fatigado $(p=0,002)$ apresentou associação estatisticamente significativa com a dor lombar (Tabela 1). Dos que se dizem mal-humorados, fatigados e sobrecarregados, 83,3\%, 70,4\%, 67,9\% têm dor lombar, respectivamente. Pelas análises ajustadas por fatores de confundimento (faixa etária, sexo, estado conjugal, turno, cargo, anos de trabalho, IMC, hora plantão, outro vínculo), o fato de se sentir mal-humorado, fatigado e sobrecarregado ao final da jornada aumentou 6,38 (IC $95 \%$ 2,00-20,33), 3,45 (IC 95\% 1,64-7,25) e 3,13 (IC $95 \% 1,62-6,05)$, respectivamente, as chances de ter dor lombar. Quanto às condições ambientais, as condições insatisfatórias do mobiliário apresentaram associação significativa com a dor lombar e aumentaram em 2,20 (IC 95\% 1,13-4,27) vezes as chances de ter dor lombar. Entre os fatores que causam insatisfação no trabalho, a falta de reconhecimento $(p=0,036)$, o ambiente de trabalho ruim $(p=0,023)$ e a sobrecarga $(p=0,000)$ foram associados à dor lombar, mas, na Regressão Logística Binária, permaneceu associada somente a sobrecarga, que aumentou em 2,69 (IC 95\% 1,41-5,13) vezes as chances de apresentar dor lombar (Tabela 1).

No que diz respeito à média geral de todas as dimensões da EACT, o valor foi 3,2, classificado como nível crítico, com condições insatisfatórias de trabalho e riscos moderados para o adoecimento. As médias de cada dimensão e a classificação de risco estão na Tabela 2.

Na dimensão "organização do trabalho", os itens "as tarefas são repetitivas" e o "ritmo de trabalho é excessivo", e na dimensão "condições de trabalho", o item "mobiliário existente no local de trabalho é inadequado" obtiveram classificação grave, o que indica um forte risco de adoecimento e requer medidas imediatas. Os únicos itens considerados satisfatórios pertencem à dimensão "relações socioprofissionais" e foram "existem dificuldades na comunicação entre chefia e subordinados", "as informações que preciso para executar minhas tarefas são de difícil acesso" e "falta apoio das chefias para o meu desenvolvimento profissional". 
Tabela 1 - Fatores associados à dor lombar inespecífica, nos últimos doze meses, em trabalhadores de enfermagem de um hospital público de Florianópolis, SC, Brasil, 2017

\begin{tabular}{|c|c|c|c|c|c|}
\hline \multirow[b]{2}{*}{ Variável } & \multicolumn{2}{|c|}{ Dor lombar } & \multirow[b]{2}{*}{ Bruta $\mathrm{OR}^{*}(\mathrm{IC})^{\dagger}$} & \multirow[b]{2}{*}{ Ajustada $\mathrm{OR}^{\ddagger}(\mathrm{IC})^{\dagger}$} & \multirow[b]{2}{*}{ p valor } \\
\hline & $\begin{array}{l}\text { Não } \\
\text { f (\%) }\end{array}$ & $\underset{f(\%)}{\operatorname{Sim}}$ & & & \\
\hline Sensação de sobrecarga & & & & & 0,001 \\
\hline Não & $109(54,5)$ & $91(45,5)$ & 1 & 1 & \\
\hline Sim & $25(32,1)$ & $53(67,9)$ & $2,53(1,46-4,40)$ & $3,13(1,62-6,05)^{\|}$ & \\
\hline Sensação de mau humor & & & & & $<0,001$ \\
\hline Não & $129(52,0)$ & $119(48,0)$ & 1 & 1 & \\
\hline Sim & $5(16,7)$ & $25(83,3)$ & $5,42(2,01-14,61)$ & $6,38(2,00-20,33)^{\|}$ & \\
\hline Sensação de fadiga & & & & & 0,002 \\
\hline Não & $118(52,7)$ & $106(47,3)$ & 1 & 1 & \\
\hline Sim & $16(29,6)$ & $38(70,4)$ & $2,64(1,39-5,01)$ & $3,45(1,64-7,25)^{\|}$ & \\
\hline Mobiliário & & & & & 0,006 \\
\hline Insatisfatório & $94(44,1)$ & $119(55,9)$ & $2,24(1,24-4,04)$ & $2,20(1,13-4,27)^{\|}$ & \\
\hline Satisfatório & $39(63,9)$ & $22(36,1)$ & 1 & 1 & \\
\hline Falta de reconhecimento & & & & & 0,036 \\
\hline Não & $57(57,0)$ & $43(43,0)$ & 1 & 1 & \\
\hline Sim & $74(43,8)$ & $95(56,2)$ & $1,70(1,03-2,80)$ & $1,78(0,99-3,20)$ & \\
\hline Ambiente ruim & & & & & 0,023 \\
\hline Não & $103(52,8)$ & $92(47,2)$ & 1 & 1 & \\
\hline Sim & $28(37,3)$ & $47(62,7)$ & $1,87(1,08-3,24)$ & $1,46(0,78-2,73)$ & \\
\hline Sobrecarga & & & & & $<0,001$ \\
\hline Não & $55(67,9)$ & $26(32,1)$ & 1 & 1 & \\
\hline Sim & $77(40,5)$ & $113(59,5)$ & $3,10(1,79-5,37)$ & $2,69(1,41-5,13)^{\|}$ & \\
\hline
\end{tabular}

*OR bruta = Análise da razão de chances (Odds Ratio) pela regressão logística univariada; ${ }^{\dagger} \mathrm{IC}=$ Intervalo com $95 \%$ de confiança; ${ }^{\ddagger}$ Ajustada OR = Análise da razão de chances (Odds Ratio) com variáveis de confusão, faixa etária, sexo, estado conjugal, turno, cargo, anos de trabalho, Índice de Massa Corpórea, hora plantão, outro vínculo com entrada no modelo pelo método enter na regressão logística multivariada; a significação do modelo pelos testes Omnibus $(p<0,05)$ e a qualidade pelo teste de Hosmer e Lemeshow $(p>0,05)$; os valores da análise dos resíduos estavam dentro do intervalo de $\pm 2,5 ;$ s $\mathrm{p}$ valor $=$ Nível de significância $p<0,05$; "Resultados da análise ajustada = Existem diferenças significativas entre as variáveis estudadas

Tabela 2 - Estatística descritiva referente às três dimensões da EACT* e classificação do risco para o adoecimento em trabalhadores de enfermagem de um hospital público de Florianópolis, SC, Brasil, 2017

\begin{tabular}{lccc}
\hline Dimensões da EACT* & Média & Desvio-padrão & Situação $^{\dagger}$ \\
\hline Organização do trabalho & 3,49 & $\pm 1,19$ & Crítico \\
Condições de trabalho & 3,56 & $\pm 1,22$ & Crítico \\
Relações socioprofissionais & 2,55 & $\pm 1,22$ & Crítico \\
\hline
\end{tabular}

*EACT = Escala de Avaliação do Contexto de Trabalho; ${ }^{+S i t u a c ̧ a ̃ o ~=~}$ Classificação de risco para o adoecimento
As dimensões "condições de trabalho" $(p=0,007)$ e "organização do trabalho" $(p=0,004)$ mostraram associação estatisticamente significativa com a dor lombar (Tabela 3). Na "organização do trabalho", a situação considerada como grave aumentou em 9,06 as chances de apresentar dor lombar; nas "condições de trabalho", a situação classificada também como grave aumentou em 3,46 vezes a chance de ter dor lombar.

Tabela 3 - Associação entre as dimensões da EACT* e dor lombar inespecífica, nos últimos 12 meses, em trabalhadores de enfermagem de um hospital público de Florianópolis, SC, Brasil, 2017

\begin{tabular}{|c|c|c|c|c|c|}
\hline \multirow[b]{2}{*}{ Variável } & \multicolumn{2}{|c|}{ Dor lombar } & \multirow[b]{2}{*}{ Bruta $\mathrm{OR}^{\dagger}(\mathrm{IC})^{\ddagger}$} & \multirow[b]{2}{*}{ Ajustada $\mathrm{OR}^{\S}(\mathrm{IC})^{\ddagger}$} & \multirow[b]{2}{*}{ p-valor" } \\
\hline & $\begin{array}{l}\text { Não } \\
\text { f (\%) }\end{array}$ & $\begin{array}{l}\text { Sim } \\
f(\%)\end{array}$ & & & \\
\hline Organização do trabalho & & & & & 0,004 \\
\hline Satisfatória & $8(80,0)$ & $2(20,0)$ & 1 & 1 & \\
\hline Crítica & $73(55,7)$ & $58(44,3)$ & $3,17(0,65-15,54)$ & $3,74(0,64-21,71)$ & \\
\hline Grave & $33(37,5)$ & $55(62,5)$ & $6,66(1,33-33,30)$ & $9,06(1,48-55,22)^{\pi}$ & \\
\hline Condições de trabalho & & & & & 0,007 \\
\hline Satisfatórias & $13(72,2)$ & $5(27,8)$ & 1 & 1 & \\
\hline Críticas & $60(54,5)$ & $50(45,5)$ & $2,16(0,72-6,49)$ & $1,63(0,48-5,52)$ & \\
\hline Graves & $47(39,2)$ & $73(60,8)$ & $4,03(1,33-12,06)$ & $3,46(1,01-11,85)^{\pi}$ & \\
\hline Relações socioprofissionais & & & & & 0,071 \\
\hline Satisfatórias & $54(58,1)$ & $39(41,9)$ & 1 & 1 & \\
\hline Críticas & $50(42,4)$ & $68(57,6)$ & $1,88(1,08-3,26)$ & $2,07(1,05-4,07)^{\pi}$ & \\
\hline Graves & $8(44,4)$ & $10(55,6)$ & $1,73(0,62-4,78)$ & $1,90(0,62-5,82)$ & \\
\hline
\end{tabular}




\section{Discussão}

Procurou-se associar a dor lombar ao processo de trabalho em uma unidade hospitalar com a amostra de 301 trabalhadores de enfermagem. A abordagem foi inovadora porque não se encontrou, na literatura, a combinação desses instrumentos para contextualizar a lombalgia ocupacional. Ressalta-se, primeiramente, a importância de pesquisas baseadas na experiência dos trabalhadores, considerando que estas geram conhecimento, informação e investigam a organização social no ambiente laboral e auxiliam no planejamento e na execução de ações que visam à prevenção de agravos à saúde(3,18).

A elevada prevalência de relatos de sintomas de dor e desconforto osteomuscular na região lombar, neste estudo, demonstra o impacto dessa problemática na equipe de enfermagem e expressa uma influência negativa na saúde desses trabalhadores. É, portanto, uma importante preocupação na prática de enfermagem e os fatores de risco das algias lombares devem ser identificados e resolvidos com prioridade ${ }^{(19)}$. Outras pesquisas nacionais e internacionais também mostraram prevalência alta de lombalgia na enfermagem com índices chegando a $85,9 \%^{(10,13,20-25)}$.

Cargas laborais como sobrecarga, fadiga e mau humor (cargas de trabalho fisiológicas e psíquicas) relacionaram-se com a dor. Altas demandas físicas ou mecânicas estressam e fatigam a musculatura e podem iniciar um processo de dor lombar pelas posições prolongadas e movimentos repetitivos. A relação entre a fadiga e a dor parece abranger lesões metabólicas e estruturais que influenciam os canais relacionados à fisiologia da dor como as glândulas basais, o tálamo, o sistema límbico e o centro cortical(26). Fatores psicossociais, como mau humor ao final da jornada e fadiga, podem influenciar a cronicidade, a frequência, a percepção e o limiar da dor(27).

A fadiga parece ter um importante papel na etiologia de sobrecargas psicofísicas. Pode ser conceituada como um desenvolvimento de uma sensação de cansaço físico e mental que modifica o estado de alerta e vigilância, afeta a capacidade para o trabalho e a execução das tarefas. Pode evoluir para uma fadiga crônica, aumentando a suscetibilidade a doenças ocupacionais. Nesse sentido, as péssimas condições de trabalho aumentam os riscos ${ }^{(28)}$. O estudo que avaliou a prevalência de desconforto osteomuscular, capacidade para o trabalho e fadiga em profissionais de enfermagem, além da alta prevalência de desconforto osteomuscular, mostrou maior fadiga e necessidade de descanso, condições estas que influenciaram diretamente a capacidade para o trabalho(28). Outro estudo constatou uma associação estatisticamente significativa entre distúrbios musculoesqueléticos e fadiga ocupacional crônica entre enfermeiros e necessidade de medidas preventivas $^{(29)}$.

Na unidade hospitalar estudada, a grande parcela dos servidores é profissional de enfermagem. Seu serviço organiza-se com centralização de decisões e divisão de tarefas entre enfermeiros e pessoal técnico de enfermagem. Algumas tarefas são individualizadas; outras são realizadas com o auxílio de outro trabalhador. Envolvem repetição de atividades e movimentos e geram os mesmos desgastes durante um tempo. Estão sujeitos a prolongamento da jornada pelo revezamento em turnos, duplo vínculo ou horas extras e expostos à grande variedade de cargas de trabalho. Justifica-se aí a multicausalidade da dor lombar. Nesse sentido, o processo de trabalho pode ser uma causa para agravos como lombalgias e outros distúrbios musculoesqueléticos (DME) relacionados ao contato com pacientes, exercício dos procedimentos e tipo de tarefa desenvolvida(30).

As dimensões da EACT "organização do trabalho" e "condições de trabalho" apresentaram associação estatisticamente significativa com a dor lombar e uma classificação crítica com moderado potencial para o adoecimento dos trabalhadores, o que demanda tomada de providências a médio e curto prazos. Resultados similares foram encontrados em estudo com trabalhadores da atenção básica(18). Outro estudo, que analisou os riscos de adoecimento do profissional de enfermagem relacionados ao contexto de trabalho em um hospital psiquiátrico, também constatou classificação crítica na dimensão "organização do trabalho", mas grave nas "condições de trabalho"(17). Quando precárias e sujeitas a várias cargas laborais, autores descrevem as condições de trabalho e sua organização como fortes fatores de adoecimento $(4,9,14,30)$ e a enfermagem está exposta a condições precárias de forma crônica(30-31). Um estudo constatou que atividades de trabalho favorecem os DME lombares e mostra a importância de um controle ocupacional com medidas organizacionais, técnicas e individuais( ${ }^{(32)}$.

Na dimensão "organização do trabalho", os itens "ritmo de trabalho excessivo" e "tarefas são repetitivas", que são cargas de trabalho fisiológicas e psíquicas, apresentaram classificação de risco grave. Estudos que analisaram os riscos de adoecimento do trabalhador de enfermagem relacionados ao contexto de trabalho em um hospital psiquiátrico encontraram classificação grave no item repetitividade de $\operatorname{tarefas}^{(17)}$ e foi o item com a pior avaliação com trabalhadores de enfermagem do serviço de hemodiálise ${ }^{(33)}$. Outro estudo revelou alta prevalência de dores lombares relacionadas a algumas tarefas, estando, entre elas, os movimentos repetitivos, 
e também constatou uma associação entre queixas lombares e absenteísmo(32). Tarefas repetidas causam cansaço e fadiga e sentimentos de tédio e raiva, que são potencializados pela pressão por produtividade e tempo para a execução das atividades ${ }^{(17,33)}$.

O ritmo de trabalho pode ser intensificado pelo prolongamento da jornada representado pelo revezamento de turnos, horas extras ou associação com outro vínculo empregatício. Apesar de, nesta pesquisa, a maioria não possuir outro emprego, o aumento da jornada está associado ao fato de fazer hora extra e plantões. Outro estudo mostrou que a carga horária diária mais longa e o grande número de pacientes atendidos aumentam os riscos laborais à saúde. O grupo com dor lombar trabalhava mais horas e permanecia mais tempo andando ou em pé, o que aumentou em $35 \%$ o risco da dor por cada hora adicional trabalhada(19). Não há limite de segurança à saúde que possa ser estabelecido quanto à extensão de uma jornada de trabalho. A luta por condições especiais, por meio da regulamentação em, no máximo, 30 horas semanais, leva a uma prática mais segura da assistência, fortalecimento da profissão, além de diminuir a exposição aos fatores de risco( ${ }^{(34)}$.

O prolongamento da jornada é a extração de mais-valia, é dispêndio excedente da força de trabalho(1,35). O capital furta, do trabalhador, horas para o desenvolvimento e a manutenção saudável do corpo, do horário das refeições e do repouso; rompe os limites morais e físicos e pode haver um esgotamento dessa força de trabalho e o encurtamento de seu tempo de vida. Para prolongar mais ainda a jornada laboral, organiza-se um revezamento de turnos, diurno e noturno ${ }^{(1,35)}$. Essa exploração leva a um desgaste físico e mental, resultado das características específicas da estratégia de extração de mais-valia, a relação entre o social e o biopsíquico(3). Entre as consequências físicas, pode estar a lombalgia ocupacional. O prolongamento da jornada também abrange a busca de economia por parte dos gestores e o aumento da produtividade. Os trabalhadores, por sua vez, ganham pagamento extra no salário, mas perdem no desgaste vivenciado pelo aumento da exposição às cargas laborais ${ }^{(34)}$.

Quanto à dimensão "condições de trabalho", o mobiliário inadequado (carga de trabalho mecânica), item com classificação crítica, potencializa os riscos ergonômicos; também destacado em outro estudo em um hospital psiquiátrico ${ }^{(17)}$ e na atenção básica ${ }^{(18)}$. Pôdese observar, na instituição estudada, inadequação em relação aos materiais, espaço físico e equipamentos. A equipe de enfermagem exerce suas atividades em espaços limitados, os armários estão posicionados de maneira inadequada, ou muito altos ou baixos, obrigando a adoção de posturas impróprias, camas com manivelas emperradas e macas sem ajustes de altura. Muitas peças do mobiliário estão deficitárias em instituições de saúde. Consequentemente, favorecem o processo de adoecimento dos trabalhadores(31). Além disso, as tarefas repetitivas, fator também com classificação de risco crítica, associadas ao mobiliário inadequado e à falta de espaço, levam ao risco ergonômico(31). A falta de materiais e equipamentos, manutenção do mobiliário e infraestrutura inadequada potencializam o ônus físico ${ }^{(14)}$.

A dimensão "relações socioprofissionais", apesar de avaliação de risco crítica, não apresentou associação estatisticamente significativa com a dor lombar. Nenhum fator obteve avaliação grave. Destacam-se os fatores "existem dificuldades na comunicação entre chefia e subordinados", "as informações que preciso para executar minhas tarefas são de difícil acesso", "falta apoio das chefias para meu desenvolvimento profissional", que foram considerados satisfatórios. Esse é um aspecto benéfico no cenário estudado porque a facilidade de comunicação e apoio exercem influência positiva no ambiente laboral. Ao contrário, a falta de apoio e integração nas relações interpessoais levam à competitividade, conflitos, desgastes emocionais e sobrecarga laboral, que podem trazer repercussões físicas desagradáveis(17). Em estudos, hospital psiquiátrico, serviço de hemodiálise e serviço móvel de urgência, as relações socioprofissionais foram avaliadas como satisfatórias ${ }^{(17,33,36)}$.

Os resultados deste estudo mostram como a organização do trabalho e suas condições podem refletir no aparecimento e manutenção de dor lombar em trabalhadores de enfermagem na instituição de saúde estudada. As pesquisas demonstram que essa é uma realidade de outros postos de trabalho em que as condições e a organização laborais podem influenciar a gênese dos agravos ${ }^{(4,31)}$.

O processo global estudado envolve muitas causas e determinantes que influenciam a relação trabalhosaúde. Procurou-se estabelecer o nexo biopsíquico, que é a expressão concreta da corporeidade humana dentro do processo histórico em determinado momento. Em alguns desses momentos, ele pode ser identificado como doença, definindo um perfil patológico. Para estabelecêlo em uma coletividade, é necessário analisar o processo de trabalho e suas condições até sua expressão no corpo dos trabalhadores ${ }^{(6)}$. A materialidade interna foi expressa pela ocorrência da lombalgia inespecífica que se manifestou pela interação entre as cargas.

Os desgastes que podem levar a agravos, apesar de adquirir corporeidade individual, devem ser considerados junto aos processos reprodutivos nas sociedades capitalistas e podem ser mensurados por sinais e sintomas inespecíficos do perfil patológico e outros indicadores ${ }^{(3,6)}$. O inter- 
relacionamento de fatores organizacionais e de condições de trabalho, neste estudo, não são riscos isolados, mas que atuam dinamicamente entre si, somam-se e potencializamse porque foram gerados sob determinadas condições ${ }^{(4,14)}$. São muitas as cargas de trabalho nesse cenário, destacandose as fisiológicas, psíquicas e mecânicas que se relacionaram com a dor lombar.

Muitos trabalhadores não têm a consciência dessa exposição, conforme demonstrado pelos resultados desta pesquisa. A inexistência de controle dos riscos ocupacionais pode levar a acidentes e doenças. Faltam, também, proteção e promoção da saúde por parte das instituições empregadoras ${ }^{(30)}$. Essa falta de percepção dos riscos não deixa de ser uma condição cômoda e desejada pelas instituições responsáveis. Por outro lado, mesmo que os trabalhadores tenham consciência dos riscos, os mesmos precisam manter lutas constantes e permanentes por melhores condições de trabalho estruturadas como prática da coletividade(30).

O desgaste envolve a perda da capacidade de desenvolver iniciativas e tomar o controle: é a negação sistemática da criatividade do trabalhador coletivo. Logo, o processo de desgaste pode interromper a luta de oposição à perda das capacidades biopsíquicas e de desenvolvimento de suas potencialidades. Por outro lado, fazer a gestão adequada de suas atividades vai deixá-los menos fragilizados, sendo a forma de os trabalhadores adquirirem o controle sobre suas próprias vidas(3). Devese olhar criteriosamente para a exposição a diversas cargas de trabalho, que podem levar ao adoecimento, para identificá-las precocemente e elaborar estratégias de prevenção, visto que também é possível reverter perdas e capacidades ${ }^{(6,9)}$.

Como limitação deste estudo, constata-se que as variáveis estudadas envolvem subjetividade e autorrelatos. Seus resultados devem ser analisados com cautela. Seriam pertinentes análises mais aprofundadas da influência da organização e das condições de trabalho na ocorrência da dor lombar, da forma como os trabalhadores analisam os resultados críticos e quais seriam as sugestões de estratégias para contornar os problemas por meio de pesquisas qualitativas. Outra limitação refere-se ao caráter transversal do estudo em que não é possível estabelecer relação entre causa e efeito. Também, o questionário NMQ possui alguns problemas na sua formatação, tais como o tipo de pergunta que inclui, além da dor, sintomas de desconforto, dormência e a falta de uma avaliação do grau de severidade dos sintomas, utilizando-se somente da sua ocorrência como a carga de risco.

No entanto, o estudo mostrou associação estatisticamente significativa entre as condições e a organização do trabalho com o desfecho dor lombar. 0 mesmo levantou aspectos fragilizados dessas dimensões que podem contribuir para o agravo no contexto de trabalho estudado e com possibilidade de estar acontecendo em outros ambientes laborais de atuação da enfermagem submetida a cargas de trabalho similares.

Contribuiu para mostrar a ocorrência da dor lombar como uma problemática de saúde desses trabalhadores a necessidade de monitoramento e vigilância. Seus resultados demonstram a importância de investir no cenário da prática da enfermagem e melhorar suas condições.

\section{Conclusão}

Nesta pesquisa, foram identificadas condições classificadas como críticas e graves no contexto laboral, expressas pela equipe de enfermagem, relacionadas às condições e à organização do trabalho. Demonstrouse que fatores como a duração da jornada, ritmos, prazos, produtividade, ambiente físico, equipamentos e instrumentos podem ter uma importante repercussão, sendo capazes de ocasionar desgastes físicos e mentais, tais como a dor lombar e outros agravos.

As dimensões "condições de trabalho" e "organização de trabalho" foram associadas à dor lombar com classificação de risco crítica e causam deficits que potencializam as cargas, destacando-se as fisiológicas, psíquicas e mecânicas.

Foi possível analisar o processo de trabalho de enfermagem hospitalar e suas articulações aos fatores relacionados à dor lombar e às consequentes implicações na saúde do trabalhador. Para isso, verificou-se que as reflexões devem ir além das causas físicas e dar visibilidade à organização e às condições de trabalho que podem gerar adoecimentos e sofrimento ao trabalhador.

Espera-se contribuir para mais conhecimentos em saúde do trabalhador referentes à dor lombar e ampliar a discussão acerca do processo de trabalho em enfermagem e fortalecimento da profissão. Novas pesquisas similares prometem contribuições relevantes à saúde e à enfermagem.

\section{Referências}

1. Peto LC, Verissimo DS. Nature and labour process in marx. Psicol Soc. [Internet]. 2018 Dec [cited Mar 07, 2019];30:e181276. Available from: http://www. scielo.br/scielo.php?script=sci_arttext\&pid=S010271822018000100248\&lng=pt\&nrm =is

2. Rocha PR, David HMSL. Determination or determinants? A debate based on the Theory on the Social Production of Health. Rev Esc Enferm USP. [Internet]. 2015 Feb [cited Dec 21, 2018];49(1):129-35. Available from: http://www.scielo.br/pdf/reeusp/v49n1/0080-6234reeusp-49-01-0129.pdf 
3. Souza KR, Rodrigues AMS, Fernandez VS, Bonfatti RJ. The health category in the perspective of worker's health: an essay about interaction, resistances and praxis. Saúde Debate. 2017 Jun;41(esp2):254-63. doi: https://doi.org/10.1590/0103-11042017S221

4. Felli VEA, Costa TF, Baptista PCP, Guimarães ALO, Anginoni BM. Exposure of nursing workers to workloads and their consequences. Rev Esc Enferm USP. 2015 Dec;49(Esp2):98-105. doi: http://dx.doi.org/10.1590/ S0080-623420150000800014

5. Pires DEP, Machado RR, Soratto J, Scherer MA, Gonçalves ASR, Trindade LL. Nursing workloads in family health: implications for universal access. Rev. Latino-Am. Enfermagem. [Internet]. 2016 Mar [cited Dec 18, 2017];24:e2677. Available from: http:// www.scielo.br/scielo.php?script=sci_arttext\&pid =S0104-11692016000100313

6. Almeida MCS, Baptista PCP, Silva A. Workloads and strain process in Community Health Agents. Rev Esc Enferm USP. [Internet]. 2016 Feb [cited Mar 8, 2019];50(1):93-100. Available from: http://www. scielo.br/scielo.php?script $=$ sci_arttext $\&$ pid $=$ S008062342016000100093\&lng=en

7. Carvalho DP, Rocha LP, Tomaschewski-Barlem JG, Barlem ELD, Cecagno D, Dalmolin GL. Productivity versus workloads in the nursing working environment. Rev Esc Enferm USP. 2017;51:e03301. doi: http://dx.doi. org/10.1590/S1980-220X2017028903301

8. Azevedo BDS, Nery AA, Cardoso JP. Occupational stress and dissatisfaction with quality of work life in nursing. Texto Contexto Enferm. 2017 Mar;26(1):e3940015. doi: http://dx.doi.org/10.1590/0104-07072017003940015

9. Karino ME, Felli VEA, Sarquis LMM, Santana LL, Silva $\mathrm{SR}$, Teixeira RC. Workloads and strain processes of nursing workers at teaching hospital. Cienc Cuid Saúde. [Internet]. 2015 Apr/Jun [cited Dec 21, 2018];14(2):1011-8. Available from: http://periodicos.uem.br/ojs/index.php/ CiencCuidSaude/article/view/21603/14751

10. Sanjoy S, Ahsan G, Nabi H, Joy Z, Hossain A. Occupational factors and low back pain: a crosssectional study of Bangladeshi female nurses. BMC Res Notes. 2017;10(1):173. doi: https://doi.org/10.1186/ s13104-017-2492-1

11. Nascimento PRC, Costa LOP. Low back pain prevalence in Brazil: a systematic review. Cad Saúde Pública. 2015 Jun;31(6):1141-56. doi: http://dx.doi. org/10.1590/0102-311X00046114

12. Hartvigsen J, Hancock MJ, Kongsted A, Louw Q, Ferreira $M L$, Genevay $S$ et al. What low back pain is and why we need to pay attention. Lancet. 2018; 391(10137):2356-67. doi: https://doi.org/10.1016/S0140-6736(18)30480-X

13. Serranheira F, Sousa-Uva M, Sousa-Uva A. Hospital nurse's tasks and work related musculoskeletal disorders symptoms: A detailed analysis. Work. [Internet]. 2015[cited Mar 08, 2019];51(3):401-9. Available from: https://www.ncbi.nlm.nih.gov/pubmed/25167914

14. Carvalho DP, Rocha LP, Barlem JGT, Specht J, Dias CDS, Schallenberger CD. Workloads and nursing workers' health: integrative review. Cogitare Enferm. 2017 Jan/Mar;22(1):1-10. doi: http://dx.doi. org/10.5380/ce.v22i1.46569

15. Siqueira K, Griep RH, Rotenberg L, Costa A, Melo E, Fonseca MJ. Interrelationships between nursing workers' state of nutrition, socio demographic factors, work and health habits. Ciênc Saúde Coletiva. [Internet]. 2015 Jun [cited Dec 19, 2018];20(6):1925-35. Available from: http://www.scielo.br/scielo.php?pid=S141381232015000601925\&script $=$ sci_abstract\&tIng $=$ pt 16. Pinheiro FA, Tróccoli BT, Carvalho CV. Validity of the Nordic Musculoskeletal Questionnaire as morbidity measurement too. Rev Saúde Pública. 2002 Jun;36(3):307-12. doi: http://dx.doi.org/10.1590/S003489102002000300008

17. Sousa KHJF, Gonçalves TS, Silva MB, Soares ECF, Nogueira MLF, Zeitoune RCG. Risks of illness in the work of the nursing team in a psychiatric hospital. Rev. Latino-Am. Enfermagem. [Internet]. 2018 Aug [cited Dec 17, 2018];26:e3032. Available from: http://dx.doi. org/10.1590/1518-8345.2458.3032

18. Maissiat GS, Lautert L, Dal Pai D, Tavares JP. Work context, job satisfaction and suffering in primary health care. Rev Gaúch Enferm. 2015 Apr/Jun;36(2):42-9. doi: http://dx.doi.org/10.1590/1983-1447.2015.02.51128 19. Shieh $\mathrm{SH}$, Sung FC, Su CH, Tsai Y, Hsieh VC. Increased low back pain risk in nurses with high workload for patient care: A questionnaire survey. Taiwan J Obstet Gynecol. 2016 Aug;55(4):525-9. doi: https://doi.org/10.1016/j. tjog.2016.06.013

20. Suliman M. Prevalence of low back pain and associated factors among nurses in Jordan. Nurs Forum. 2018;53:425-31. doi: https://doi.org/10.1111/nuf.12269 21. Santos EC, Andrade RD, Lopes SGR, Valgas C. Prevalence of musculoskeletal pain in nursing professionals working in orthopedic setting. Rev Dor. [Internet]. 2017 Dec [cited Mar 8, 2019];18(4):298-306. Available from: http:// www.scielo.br/scielo.php?script=sci_arttext\&pid=S180600132017000400298\&lng=en

22. Sharma S, Shrestha N, Jensen MP. Pain-related factors associated with lost work days in nurses with low back pain: across-sectional study. Scand J Pain. 2016;11:3641. doi: https://doi.org/10.1016/j.sjpain.2015.11.007

23. Sezgin D, Esin MN. Predisposing factors for musculoskeletal symptoms in intensive care unit nurses. Int Nurs Rev. 2015 Dec;62(1):92-101. doi: https://doi. org/10.1111/inr.12157

24. Skela-Savic B, Pesjak K, Hvalic-Touzery S. Low back pain among nurses in Slovenian hospitals: cross- 
sectional study. Int Nurs Rev. 2017 Apr;26:1-8. doi: https://doi.org/10.1111/inr.12376

25. Boughattas W, El Maalel O, Maoua M, Bougmiza I, Kalboussi $H$, Brahem $A$ et al. Low back pain among nurses: prevalence, and occupational risk factors. Occup Environ Med. 2017 Feb;5(1),26- 37. doi: https://doi. org/10.4236/odem.2017.51003

26. Santos JKV, Gomes Junior VFF, Souza AS, Farias NS, Marques SS, Costa JM. Socio-demographic and physical-functional profile of low back pain patients assisted in Manaus-AM. Rev Dor. [Internet]. 2015 Oct/ Dec [cited Aug 18, 2018];16(4):272-5. Available from: http://www.scielo.br/scielo.php?script=sci_arttext\&pid $=$ S1806-00132015000400272

27. Bonzini M, Bertu'L, Veronesi G, Conti M, Coggon D, Ferrario MM. Is muskuloeskeletal pain a consequence or a cause of occupational stress? A longitudinal study. Int Arch Occup Environ Health. 2015 Sep;88(5):607-12. doi: https://doi.org/10.1007/s00420-014-0982-1

28. Silva TPD, Araújo WN, Stival MM, Toledo AM, Burke TN, Carregaro RL. Musculoskeletal discomfort, work ability and fatigue in nursing professionals working in a hospital environment. Rev Esc Enferm USP. 2018;52:e03332. doi: http://dx.doi.org/10.1590/S1980-220X2017022903332 29. Younan L, Clinton M, Fares S, El Jardali F, Samaha H. The relationship between work-related musculoskeletal disorders, chronic occupational fatigue, and work organization: a multi-hospital cross-sectional study. J Adv Nurs. 2019 Jan;00:1-11. doi: https://doi.org/10.1111/ jan. 13952

30. Shoji S, Souza NVDO, Farias SNP. Impact of workplace on the health of nursing professionals at a specialized outpatient clinic. Reme: Rev Min Enferm. 2015 Jan/ Mar;19(1):43-8. doi: http://dx.doi.org/10.5935/14152762.20150004

31. Shoji S, Souza NVDO, Farias SNP, Vieira MLC, Progianti JM. Proposals for improving working conditions at an outpatient clinic: the nursing standpoint. Esc Anna Nery. 2016 Apr/Jun;20(2):303-9. doi: http://dx.doi. org/10.5935/1414-8145.20160041

32. Ribeiro T, Serranheira F, Loureiro H. Work related musculoskeletal disorders in primary health care nurses. Appl Nurs Res. 2017 Feb;33:72-7. doi: https://doi. org/10.1016/j.apnr.2016.09.003
33. PrestesFC, BeckCLC, Magnago TSBS, Silva RM, Tavares JP. Working context in a hemodialysis service: evoluation of nursing staff. Texto Contexto Enferm. [Internet]. 2015 Jul/Sep [cited Dec 18, 2018];24(3):637-45. Available from: http://www.scielo.br/scielo.php?script=sci_arttext \&pid=S0104-07072015000300637

34. Silva JA Neto, Torres CRD, Feitosa KVAF, Gouveia MTO, Torres JRD. Legal aspects of work shift in nursing: theoretical reflection. Rev Enferm UFPI. 2015 Jun/ Sep;4(3):95-8. doi: http://dx.doi.org/10.26694/reufpi. v4i3.2368

35. Alves JCL, Jackson JM Filho. Work, health, and political training in marx's worker's inquiry. Trab Educ Saúde.[Internet]. 2017 [cited Mar 09, 2019]:15(1):13-31. Available from: http://www. scielo.br/scielo.php?script=sci_arttext\&pid=S198177462017000100013\&lng =en\&nrm =iso

36. Worm FA, Pinto MAO, Schiavenato D, Ascari RA, Trindade LL, Silva OM. Risk of disease of nursing professionals at work in emergency mobile service. Rev Cuidarte. 2016;7(2):1288-96. doi: http://dx.doi. org/10.15649/cuidarte.v7i2.329 